Communications in Physics, Vol. 25, No. 4 (2015), pp. 291-298

DOI:10.15625/0868-3166/25/4/7651

\title{
INVERTED NEUTRINO MASS HIERARCHY AND MIXING IN THE ZEE-BABU MODEL
}

\author{
VO VAN VIEN
}

Department of Physics, Tay Nguyen University, 567 Le Duan, Buon Ma Thuot, DakLak, Vietnam

HOANG NGOC LONG

Institute of Physics, VAST, 10 Dao Tan, Ba Dinh, Hanoi, Vietnam

PHAM NGOC THU

Faculty of Mathematics-Physics-Informatics, Tay Bac University, Son La city, Vietnam

E-mail: wvienk16@gmail.com

Received 29 November 2015

Accepted for publication 24 December 2015

\begin{abstract}
We show that the neutrino mass matrix of the Zee-Babu model is able to fit the recent data on neutrino masses and mixing with non-zero $\theta_{13}$ in the inverted neutrino mass hierarchy. The results show that the Majorana phases are equal to zero and the Dirac phase $(\delta)$ is predicted to either 0 or $\pi$, $i$. e, there is no CP violation in the Zee-Babu model at the two loop level. The effective mass governing neutrinoless double beta decay and the sum of neutrino masses are consistent with the recent analysis.

Keywords: neutrino mass and mixing, non-standard-model neutrinos, Zee-Babu model.
\end{abstract}

\section{INTRODUCTION}

At present, neutrino and Higgs physics are hot topics in Particle Physics. The neutrino mass and mixing are the first evidence of beyond Standard Model (SM) physics. Despite the Higgs boson has been discovered by the ATLAS [1] and the CMS [2] but to which model it belongs still an open question. For the aforementioned reasons, the search for an extended model coinciding with the current data on neutrino physics is one of our top priorities. In our opinion, the model with the simplest particle content is preferred. By this criterion, the Zee-Babu model [3-5] is very attractive. In our previous work [6], we have derived the exact solution for the neutrino mass matrix in the model under consideration and derived some regions of the parameters in the normal neutrino mass hierarchy.

As far as we know at present the values of the absolute neutrino masses as well as the mass ordering of neutrinos are still an open problem. The mass ordering of neutrino depends on the sign of $\Delta m_{31}^{2}$ which is currently unknown. In the case of 3-neutrino mixing, the two possible signs of $\Delta m_{31}^{2}$ corresponding to two types of neutrino mass spectrum can be provided as follows

(1) Normal hierarchy (NH): $\left|m_{1}\right| \simeq\left|m_{2}\right|<\left|m_{3}\right|, \Delta m_{31}^{2}=m_{3}^{2}-m_{1}^{2}>0$.

(C)2015 Vietnam Academy of Science and Technology 
(2) Inverted hierarchy (IH): $\left|m_{3}\right|<\left|m_{1}\right| \simeq\left|m_{2}\right|, \Delta m_{31}^{2}=m_{3}^{2}-m_{1}^{2}<0$.

In this paper, we focus on the effective mass governing neutrinoless double beta decay and the sum of neutrino masses. As will be discussed below, the model can give some regions of the parameters where neutrino mixing angles and the inverted neutrino mass hierarchy obtained consistent with the recent experimental data. Indeed, by starting from the neutrino mass matrix in the Zee-babu model [3-5], we get the exact solution, i.e., the eigenstates and the eigenvalues. Comparing the model results with the experimental data we get the model parameters. The effective mass governing neutrinoless double beta decay and the sum of neutrino mass are consistent with the recent analysis.

This paper is organized as follows. In Sec. II, we briefly present the Zee-Babu model and its neutrino mass matrix. Sec. III is devoted for the solution and phenomenology with focus on the inverted spectrum. We summarize our result in the last section - Sec. IV.

\section{ZEE-BABU MODEL AND NEUTRINO MASS MATRIX}

With just two $\mathrm{SU}(2)_{L}$ singlet Higgs fields, a singly charged field $h^{-}$and a doubly charged field $k^{--}$and without right-handed neutrinos, the new Yukawa interactions in the Zee-Babu model [4] are

$$
L_{Y}=f_{a b} \overline{\left(\psi_{a L}\right)^{C}} \psi_{b L} h^{+}+h_{a b}^{\prime} \overline{\left(l_{a R}\right)^{C}} l_{b R} k^{++}+H . c .,
$$

where $\psi_{L}$ stands for the left-handed lepton doublet, $l_{R}$ for the right-handed charged lepton singlet and $(a, b=e, \mu, \tau)$ being the generation indices, a superscript ${ }^{C}$ indicating charge conjugation. Note that $f_{a b}$ is antisymmetric $\left(f_{a b}=-f_{b a}\right)$ and $h_{a b}^{\prime}$ is symmetric $\left(h_{a b}^{\prime}=h_{b a}^{\prime}\right)$. In terms of the component fields, the interaction Lagrangian is given by

$$
\begin{aligned}
\mathscr{L}_{Y} & =2\left[f_{e \mu}\left(\bar{v}_{e}^{c} \mu_{L}-\bar{v}_{\mu}^{c} e_{L}\right)+f_{e \tau}\left(\bar{v}_{e}^{c} \tau_{L}-\bar{v}_{\tau}^{c} e_{L}\right)+f_{\mu \tau}\left(\overline{v_{\mu}^{c}} \tau_{L}-\bar{v}_{\tau}^{c} \mu_{L}\right)\right] h^{+} \\
& +\left[h_{e e} \bar{e}^{c} e_{R}+h_{\mu \mu} \bar{\mu}^{c} \mu_{R}+h_{\tau \tau} \bar{\tau}^{c} \tau_{R}+h_{e \mu} \bar{e}^{c} \mu_{R}+h_{e \tau} \bar{e}^{\bar{c}} \tau_{R}+h_{\mu \tau} \bar{\mu}^{c} \tau_{R}\right] k^{++} \\
& + \text {H.c. }
\end{aligned}
$$

where we have used $h_{a a}=h_{a a}^{\prime}, h_{a b}=2 h_{a b}^{\prime}$ for $a \neq b$. In Eq. (1), the lepton number is conserved, and neutrino mass will be generated due to the Higgs potential given by:

$$
V\left(\phi, h^{+}, k^{++}\right)=\mu\left(h^{-} h^{-} k^{++}+h^{+} h^{+} k^{--}\right)+\cdots .
$$

Here, the lepton number is violated by two units, hence one expects the Majorana neutrino masses. From Eq.(1), it follows both $h^{-}$and $k^{--}$carry lepton number two, so the coefficient $\mu$ in (3) also carries lepton number two. Therefore it is expected that the Majorana neutrino masses are generated by loop quantum effects. At the two-loop level, the mass matrix for Majorana neutrinos is given by

$$
M_{a b}=8 \mu f_{a c} h_{c d}^{*} m_{c} m_{d} I_{c d}\left(f^{+}\right)_{d b},
$$

where $I_{c d}$ has the form [7]

$$
\begin{aligned}
I_{c d} & =\int \frac{d^{4} k}{(2 \pi)^{4}} \int \frac{d^{4} q}{(2 \pi)^{4}} \frac{1}{k^{2}-m_{c}^{2}} \frac{1}{k^{2}-M_{h}^{2}} \frac{1}{q^{2}-m_{d}^{2}} \\
& \times \frac{1}{q^{2}-M_{h}^{2}} \frac{1}{(k-q)^{2}-M_{k}^{2}} .
\end{aligned}
$$


Assuming masses of new Higgses are much larger than lepton ones, we can evaluate $I_{c d}$ as follows

$$
I_{c d} \simeq I=\frac{1}{\left(16 \pi^{2}\right)^{2}} \frac{1}{M^{2}} \frac{\pi^{2}}{3} \tilde{I}(r), M \equiv \max \left(M_{k}, M_{h}\right) .
$$

Here $\tilde{I}(r)$ is a function of the ratio of the masses of the charged Higgses $r \equiv M_{k}^{2} / M_{h}^{2}$,

$$
\tilde{I}(r)= \begin{cases}1+\frac{3}{\pi^{2}}\left(\log ^{2} r-1\right) & \text { for } r \gg 1 \\ 1 & \text { for } r \rightarrow 0\end{cases}
$$

which is close to 1 for a wide range of scalar masses.

The neutrino mass matrix in (4) is symmetric and given by [8]

$$
\begin{gathered}
\mathscr{M}_{v}=-I \mu f_{\mu \tau}^{2} \times \\
\left(\begin{array}{ccc}
\varepsilon^{2} \omega_{\tau \tau}+2 \varepsilon \varepsilon^{\prime} \omega_{\mu \tau}+\varepsilon^{\prime 2} \omega_{\mu \mu} & \varepsilon \omega_{\tau \tau}+\varepsilon^{\prime}\left(\omega_{\mu \tau}-\varepsilon \omega_{e \tau}-\varepsilon^{\prime} \omega_{e \mu}\right) & -\varepsilon^{\prime} \omega_{\mu \mu}-\varepsilon\left(\omega_{\mu \tau}+\varepsilon \omega_{e \tau}+\varepsilon^{\prime} \omega_{e \mu}\right) \\
\star & \omega_{\tau \tau}+\varepsilon^{\prime 2} \omega_{e e}-2 \varepsilon^{\prime} \omega_{e \tau} & \varepsilon \varepsilon^{\prime} \omega_{e e}-\omega_{\mu \tau}-\varepsilon \omega_{e \tau}+\varepsilon^{\prime} \omega_{e \mu} \\
\star & \star & \omega_{\mu \mu}+2 \varepsilon \omega_{e \mu}+\varepsilon^{2} \omega_{e e}
\end{array}\right)
\end{gathered}
$$

where we have redefined parameters:

$$
\varepsilon \equiv \frac{f_{e \tau}}{f_{\mu \tau}}, \quad \varepsilon^{\prime} \equiv \frac{f_{e \mu}}{f_{\mu \tau}} \quad \omega_{a b} \equiv m_{a} h_{a b}^{*} m_{b}
$$

Let us denote $[6,9]$

$$
\begin{aligned}
\omega_{\tau \tau}^{\prime} & \equiv \omega_{\tau \tau}+\varepsilon^{\prime 2} \omega_{e e}-2 \varepsilon^{\prime} \omega_{e \tau} \\
\omega_{\mu \tau}^{\prime} & \equiv \omega_{\mu \tau}+\varepsilon \omega_{e \tau}-\varepsilon^{\prime} \omega_{e \mu}-\varepsilon \varepsilon^{\prime} \omega_{e e} \\
\omega_{\mu \mu}^{\prime} & \equiv \omega_{\mu \mu}+2 \varepsilon \omega_{e \mu}+\varepsilon^{2} \omega_{e e}
\end{aligned}
$$

then the neutrino mass matrix can be rewritten in the compact form

$$
\mathscr{M}_{v}=-I \mu f_{\mu \tau}^{2}\left(\begin{array}{ccc}
\varepsilon^{2} \omega_{\tau \tau}^{\prime}+2 \varepsilon \varepsilon^{\prime} \omega_{\mu \tau}^{\prime}+\varepsilon^{\prime 2} \omega_{\mu \mu}^{\prime} & \varepsilon \omega_{\tau \tau}^{\prime}+\varepsilon^{\prime} \omega_{\mu \tau}^{\prime} & -\varepsilon \omega_{\mu \tau}^{\prime}-\varepsilon^{\prime} \omega_{\mu \mu}^{\prime} \\
\star & \omega_{\tau \tau}^{\prime} & -\omega_{\mu \tau}^{\prime} \\
\star & \star & \omega_{\mu \mu}^{\prime}
\end{array}\right) .
$$

Next we turn to solution and implication to current neutrino data with a rather large $\theta_{13}$.

\section{SOLUTION AND PHENOMENOLOGY}

To begin this section, let us present the recent data on neutrino mass and mixing. The best fit values of neutrino mass squared differences and the leptonic mixing angles in [10] have been given to be slightly deviation from Tri-bimaximal mixing form in the inverted spectrum, as shown in Tab. 1 with a rather large $\theta_{13}$.

The matrix $\mathscr{M}_{v}$ in (10) has three exact eigenvalues given by

$$
\begin{aligned}
\lambda_{1} & =0, \\
\lambda_{2,3} & =\frac{1}{2}\left(-k F \pm \sqrt{k^{2}\left[F^{2}+4\left(1+\varepsilon^{2}+\varepsilon^{\prime 2}\right)\left(\omega_{\mu \tau}^{\prime 2}-\omega_{\mu \mu}^{\prime} \omega_{\tau \tau}^{\prime}\right)\right]}\right),
\end{aligned}
$$


Table 1. The experimental values of neutrino mass squared splittings and leptonic mixing parameters, taken from [10] for inverted hierarchy.

\begin{tabular}{llll}
\hline Parameter & Best fit & $1 \sigma$ range & $2 \sigma$ range \\
\hline$\Delta m_{21}^{2}\left(10^{-5} \mathrm{eV}^{2}\right)$ & 7.62 & $7.43-7.81$ & $7.27-8.01$ \\
$\Delta m_{13}^{2}\left(10^{-3} \mathrm{eV}^{2}\right)$ & 2.43 & $2.37-2.50$ & $2.29-2.58$ \\
$\sin ^{2} \theta_{12}$ & 0.32 & $0.303-0.336$ & $0.29-0.35$ \\
$\sin ^{2} \theta_{23}$ & 0.60 & $0.569-0.626$ & $0.39-0.65$ \\
$\sin ^{2} \theta_{13}$ & 0.025 & $0.0223-0.0276$ & $0.02-0.03$ \\
\hline
\end{tabular}

where we have denoted

$$
k=\mu I f_{\mu \tau}^{2}, \quad F=\left(1+\varepsilon^{\prime 2}\right) \omega_{\mu \mu}^{\prime}+2 \varepsilon \varepsilon^{\prime} \omega_{\mu \tau}^{\prime}+\left(1+\varepsilon^{2}\right) \omega_{\tau \tau}^{\prime} .
$$

The massless eigenstate is given by

$$
v_{1}=\frac{1}{\sqrt{f_{e \mu}^{2}+f_{e \tau}^{2}+f_{\mu \tau}^{2}}}\left(f_{\mu \tau} v_{e}-f_{e \tau} v_{\mu}+f_{e \mu} v_{\tau}\right)
$$

Until now values of neutrino masses (or the absolute neutrino masses) as well as the mass ordering of neutrinos are unknown. An upper bound on the absolute value of neutrino mass was found from the analysis of the cosmological data [11]

$$
m_{i} \leq 0.6 \mathrm{eV}
$$

while the upper limit on the sum of neutrino mass is given in Ref. [12]

$$
\sum_{i=1}^{3} m_{i} \leq 0.66 \mathrm{eV}
$$

In the inverted hierarchy, three neutrino masses are chosen as follows:

$$
m_{1}=\lambda_{3}, m_{2}=\lambda_{2}, m_{3}=0,
$$

with $\lambda_{i}(i=1,2,3)$ is defined in (11), and the corresponding eigenstates put in the neutrino mixing matrix:

$$
U_{v I}=\left(\begin{array}{ccc}
\frac{A_{2}}{\sqrt{1+A_{2}^{2}+B_{2}^{2}}} & -\frac{A_{1}}{\sqrt{1+A_{1}^{2}+B_{1}^{2}}} & \frac{1}{\sqrt{1+\varepsilon^{2}+\varepsilon^{\prime 2}}} \\
\frac{B_{2}}{\sqrt{1+A_{2}^{2}+B_{2}^{2}}} & -\frac{B_{1}}{\sqrt{1+A_{1}^{2}+B_{1}^{2}}} & -\frac{\varepsilon}{\sqrt{1+\varepsilon^{2}+\varepsilon^{\prime 2}}} \\
\frac{1}{\sqrt{1+A_{2}^{2}+B_{2}^{2}}} & -\frac{1}{\sqrt{1+A_{1}^{2}+B_{1}^{2}}} & \frac{\varepsilon^{\prime}}{\sqrt{1+\varepsilon^{2}+\varepsilon^{\prime 2}}}
\end{array}\right),
$$

where [6]

$$
\begin{aligned}
A_{1,2} & =\frac{-k\left[\varepsilon\left(\varepsilon^{2}-1\right) \omega_{\mu \mu}^{\prime}+2 \varepsilon^{\prime}\left(1+\varepsilon^{2}\right) \omega_{\mu \tau}^{\prime}+\varepsilon\left(1+\varepsilon^{2}\right) \omega_{\tau \tau}^{\prime}\right] \pm \varepsilon \sqrt{k^{2} F^{\prime}}}{2 k\left[\varepsilon \varepsilon^{\prime} \omega_{\mu \mu}^{\prime}+\left(1+\varepsilon^{2}\right) \omega_{\mu \tau}^{\prime}\right]} \\
B_{1,2} & \equiv \frac{k\left(1+\varepsilon^{\prime 2}\right) \omega_{\mu \mu}^{\prime}-k\left(1+\varepsilon^{2}\right) \omega_{\tau \tau}^{\prime} \pm \sqrt{k^{2} F^{\prime}}}{2 k\left[\varepsilon \varepsilon^{\prime} \omega_{\mu \mu}^{\prime}+\left(1+\varepsilon^{2}\right) \omega_{\mu \tau}^{\prime}\right]}
\end{aligned}
$$


and

$$
F^{\prime}=F^{2}+4\left(1+\varepsilon^{2}+\varepsilon^{\prime 2}\right)\left(\omega_{\mu \tau}^{\prime 2}-\omega_{\mu \mu}^{\prime} \omega_{\tau \tau}^{\prime}\right)
$$

The eigenstates $v_{i}$ corresponding to the eigenvalues $m_{i}(i=1,2,3)$ are found to be

$$
\begin{aligned}
& v_{1}=\frac{A_{2}}{\sqrt{1+A_{2}^{2}+B_{2}^{2}}} v_{e}+\frac{B_{2}}{\sqrt{1+A_{2}^{2}+B_{2}^{2}}} v_{\mu}+\frac{1}{\sqrt{1+A_{2}^{2}+B_{2}^{2}}} v_{\tau}, \\
& v_{2}=-\frac{A_{1}}{\sqrt{1+A_{1}^{2}+B_{1}^{2}}} v_{e}-\frac{B_{1}}{\sqrt{1+A_{1}^{2}+B_{1}^{2}}} v_{\mu}-\frac{1}{\sqrt{1+A_{1}^{2}+B_{1}^{2}}} v_{\tau}, \\
& v_{3}=\frac{1}{\sqrt{f_{e \mu}^{2}+f_{e \tau}^{2}+f_{\mu \tau}^{2}}}\left(f_{\mu \tau} v_{e}-f_{e \tau} v_{\mu}+f_{e \mu} v_{\tau}\right) .
\end{aligned}
$$

Some useful relations are in order [6]

$$
\begin{aligned}
A_{1} A_{2}+B_{1} B_{2}+1 & =0 \\
A_{1}-\varepsilon B_{1}+\varepsilon^{\prime} & =0 \\
A_{2}-\varepsilon B_{2}+\varepsilon^{\prime} & =0 \\
\left(A_{1}-A_{2}\right) /\left(B_{1}-B_{2}\right) & =\varepsilon .
\end{aligned}
$$

One also has

$$
\begin{aligned}
A_{1} A_{2} & =\frac{\left(\varepsilon^{\prime 2}-\varepsilon^{2}\right) \omega_{\mu \tau}^{\prime}+\varepsilon \varepsilon^{\prime}\left(\omega_{\tau \tau}^{\prime}-\omega_{\mu \mu}^{\prime}\right)}{\varepsilon \varepsilon^{\prime} \omega_{\mu \mu}^{\prime}+\left(1+\varepsilon^{2}\right) \omega_{\mu \tau}^{\prime}} \\
B_{1} B_{2} & =-\frac{\left(1+\varepsilon^{\prime 2}\right) \omega_{\mu \tau}^{\prime}+\varepsilon \varepsilon^{\prime} \omega_{\tau \tau}^{\prime}}{\varepsilon \varepsilon^{\prime} \omega_{\mu \mu}^{\prime}+\left(1+\varepsilon^{2}\right) \omega_{\mu \tau}^{\prime}}
\end{aligned}
$$

In the standard Particle Data Group (PDG) parametrization, the neutrino mixing matrix $\left(U_{P M N S}\right)$ can be parametrized as [13]

$$
U_{P M N S}=\left(\begin{array}{ccc}
c_{12} c_{13} & s_{12} c_{13} & s_{13} e^{-i \delta} \\
-s_{12} c_{23}-c_{12} s_{23} s_{13} e^{i \delta} & c_{12} c_{23}-s_{12} s_{23} s_{13} e^{i \delta} & s_{23} c_{13} \\
s_{12} s_{23}-c_{12} c_{23} s_{13} e^{i \delta} & -c_{12} s_{23}-s_{12} c_{23} s_{13} e^{i \delta} & c_{23} c_{13}
\end{array}\right) \times P
$$

where $P=\operatorname{diag}\left(1, e^{i \frac{\alpha_{21}}{2}}, e^{i \frac{\alpha_{31}}{2}}\right)$, and $c_{i j}=\cos \theta_{i j}, s_{i j}=\sin \theta_{i j}$ with $\theta_{12}, \theta_{23}$ and $\theta_{13}$ being the solar, atmospheric and the reactor angles, respectively, the angles $\theta_{i j}=\left[0, \frac{\pi}{2}\right] . \delta=[0,2 \pi]$ is the Dirac $\mathrm{CP}$ violation phase and $\alpha_{21}, \alpha_{31}$ are two Majorana $\mathrm{CP}$ violation phases. It is to be mentioned that in our previous work [6], the Majorana has been included in a simple form. 
By comparing Eqs. (17) and (24), all the parameters in the lepton mixing matrix in (17) can be parameterized in terms of three Euler's angles $\theta_{i j}$ as follows:

$$
\begin{aligned}
\varepsilon & =\frac{c_{13}\left(\frac{B_{2}}{\sqrt{1+A_{2}^{2}+B_{2}^{2}}}+s_{12} c_{23}\right)}{c_{12} s_{13}^{2}}, \varepsilon^{\prime}=-\frac{c_{23}}{s_{23}} \cdot \varepsilon, \\
e^{i \frac{\alpha_{21}}{2}} & =-\frac{1}{s_{12} c_{13}} \frac{A_{1}}{\sqrt{1+A_{1}^{2}+B_{1}^{2}}}, \\
e^{i \frac{\alpha_{31}}{2}} & =-\frac{1}{c_{13} s_{23}} \frac{\varepsilon}{\sqrt{1+\varepsilon^{2}+\varepsilon^{\prime 2}}}, \\
e^{i \delta} & =-\frac{s_{13}}{c_{13} s_{23}} \varepsilon,
\end{aligned}
$$

and two solutions with $A_{2}, B_{2}$ :

$$
\begin{aligned}
& A_{2}=\frac{c_{12} c_{13}}{s_{12} s_{23}+c_{12} s_{13} c_{23}} \equiv A_{2}^{+}, \\
& B_{2}=\frac{c_{12} s_{12} c_{13}+s_{23} c_{23}\left(c_{12}^{2} c_{13}^{2}-1\right)}{s_{12}^{2}+c_{23}^{2}\left(c_{12}^{2} c_{13}^{2}-1\right)} \equiv B_{2}^{+},
\end{aligned}
$$

or

$$
\begin{aligned}
& A_{2}=\frac{c_{12} s_{12} c_{13}}{s_{12}^{2} s_{23}-c_{12} s_{12} s_{13} c_{23}} \equiv A_{2}^{-}, \\
& B_{2}=\frac{-c_{12} s_{12} c_{13}+s_{23} c_{23}\left(c_{12}^{2} c_{13}^{2}-1\right)}{s_{12}^{2}+c_{23}^{2}\left(c_{12}^{2} c_{13}^{2}-1\right)} \equiv B_{2}^{-} .
\end{aligned}
$$

Let us consider both the solution in Eqs. (27) and (28).

\section{III.1. The solution with $A_{2}^{+}, B_{2}^{+}$}

It is easily shown that, in this case, the model is consistent because the five experimental constraints on the mixing angles and squared mass differences of neutrinos can be respectively fitted with all parameters of the model. Indeed, with $A_{2}=A_{2}^{+}, B_{2}=B_{2}^{+}$given in Eq. (27), taking the data in Ref. [10] given in Tab. 1, we obtain

$$
\begin{aligned}
A_{1} & =-0.95944, \quad A_{2}=1.56394, \quad B_{1}=-1.01484, \quad B_{2}=-0.49319 \\
\varepsilon & =4.83735, \quad \varepsilon^{\prime}=-3.94968, \quad \frac{\varepsilon}{\varepsilon^{\prime}}=-1.22474 \\
e^{i \frac{\alpha_{21}}{2}} & =1, \quad e^{i \frac{\alpha_{31}}{2}}=-1, \quad e^{i \delta}=-1
\end{aligned}
$$

Eq. (31) implies $\alpha_{21}=0, \alpha_{31}=2 \pi, \delta=\pi$, i.e, there is no CP-violation. The neutrino mixing matrix then takes the form:

$$
U_{v I}=\left(\begin{array}{ccc}
0.81425 & 0.55857 & 0.15811 \\
-0.25678 & 0.59082 & -0.76485 \\
0.52064 & -0.58218 & -0.62450
\end{array}\right)
$$


The physical neutrino masses are obtained as

$$
\begin{aligned}
& m_{1}=\sqrt{\Delta m_{13}^{2}}=4.9295 \times 10^{-2} \mathrm{eV}, \\
& m_{2}=\sqrt{m_{1}^{2}-\Delta m_{21}^{2}}=4.8516 \times 10^{-2} \mathrm{eV}, m_{3}=0,
\end{aligned}
$$

and the effective masses $\left\langle m_{e e}\right\rangle, m_{\beta}$ governing neutrinoless double beta decay [14-18] as well as the sum of the neutrino masses are given by:

$$
\begin{aligned}
& \left\langle m_{e e}\right\rangle=\left|\sum_{i=1}^{3} U_{e i}^{2} m_{i}\right|=0.04782 \mathrm{eV} \\
& m_{\beta}=\sum_{i=1}^{3}\left|U_{e i}\right|^{2} m_{i}^{2}=0.04843 \mathrm{eV} \\
& m_{1}+m_{2}+m_{3}=0.09781 \mathrm{eV}
\end{aligned}
$$

As before, we assume $\omega_{\mu \mu}^{\prime}=\omega_{\tau \tau}^{\prime}=\omega^{\prime}[6]$. Substituting $\varepsilon, \varepsilon^{\prime}$ in Eq. (30) into (11) and (16) yields

$$
\omega_{\mu \tau}^{\prime}=1.07194 \omega^{\prime}, k=-\frac{0.0200278}{\omega^{\prime}},
$$

or

$$
\omega_{\mu \tau}^{\prime}=1.07399 \omega^{\prime}, k=-\frac{0.0197385}{\omega^{\prime}} .
$$

Eq. (31) shows that in this case one the Dirac and one Majorana phase is nonzero, however, there is no CP violation phase. Our next step is the second case.

\section{III.2. The solution with $A_{2}^{-}, B_{2}^{-}$}

In this case, taking the data in [10] given in Tab. 1, we obtain

$$
\begin{aligned}
& A_{1}=-0.80333, \quad A_{2}=2.28904, \quad B_{1}=-0.65043, \quad B_{2}=-1.2897, \\
& \varepsilon=-4.83735, \quad \varepsilon^{\prime}=3.94968, \quad \frac{\varepsilon}{\varepsilon^{\prime}}=-1.22474, \\
& e^{i \frac{\alpha_{21}}{2}}=e^{i \frac{\alpha_{31}}{2}}=e^{i \delta}=1 .
\end{aligned}
$$

Eq. (41) implies $\alpha_{21}=\alpha_{31}=\delta=0$. Then, the neutrino mixing matrix is

$$
U_{v I}=\left(\begin{array}{ccc}
0.81425 & 0.55857 & 0.15811 \\
-0.45877 & 0.45225 & 0.76485 \\
0.35572 & -0.69532 & 0.6245
\end{array}\right)
$$

Three neutrino masses are given in (36), and the effective masses $\left\langle m_{e e}\right\rangle, m_{\beta}$ governing neutrinoless double beta decay as well as the sum of the neutrino masses are given by (34), (35) and (36). The relation between $\omega_{\mu \tau}^{\prime}, k$ and $\omega^{\prime}$ are given in Eqs.(37) and (38). Note that in this case, all Dirac and Majorana violation phases are vanished. 


\section{CONCLUSION}

In this paper we have derived the exact eigenvalues and eigenstates of the neutrino mass matrix in the Zee-Babu model in which the most recent data on neutrino masses and mixing with large $\theta_{13}$ are updated. For the inverted spectrum, one phase $\left(\alpha_{31}\right)$ takes the value $2 \pi$ and the Dirac phase $(\delta)$ is predicted to either 0 or $\pi$, i. e, there is no CP violation in the Zee-Babu model at the two loop level. Taking into account of the effective mass governing neutrinoless double beta decay and the sum of neutrino, we have showed that this model fits well with the recent experimental data in inverted spectrum. Therefore we conclude that the Zee-Babu model is fascinating one for neutrino physics.

\section{ACKNOWLEDGMENTS}

This research has received funding from the Vietnam National Foundation for Science and Technology Development (NAFOSTED) under grant number 103.01-2014.51.

\section{REFERENCES}

[1] ATLAS Collaboration, G. Aad et al., Phys. Lett. B 716 (2012) 1, arXiv: 1207.7214 [hep-ex].

[2] CMS Collaboration, S. Chatrchyan et al., Phys. Lett. B 716 (2012) 30, arXiv: 1207.7235 [hep-ex].

[3] A. Zee, Phys. Lett. B 93 (1980) 389.

[4] A. Zee, Nucl. Phys. B 264 (1986) 99.

[5] K. S. Babu, Phys. Lett. B 203 (1988) 132.

[6] Hoang Ngoc Long and Vo Van Vien, Int. J. Mod. Phys. A 29 (2014) 1450072, arXiv:1405.1622 [hep-ph].

[7] K. L. McDonald and B. H. J. McKellar (2003), Evaluating The Two Loop Diagram Responsible For Neutrino Mass In Babu's Model, arXiv: 0309270 [hep- ph].

[8] K. S. Babu, C. Macesanu, Phys. Rev. D 67 (2003) 073010, arXiv: 0212058 [hep-ph].

[9] T. Araki and C. Q. Geng, Phys. Lett. B 694 (2010) 113.

[10] D. V. Forero, M. Tortola and J. W. F. Valle, Phys. Rev. D 86 (2012) 073012, arXiv:1205.4018 [hep-ph].

[11] M. Tegmark et al, Phys. Rev. D 69 (2004) 103501.

[12] P. A. R. Ade et al. [Planck Collaboration], Astron.Astrophys. 571 (2014) A16, arXiv:1303.5076 [astro-ph.CO].

[13] J. Beringer, et al., Review of Particle Physics (Particle Data Group), Phys. Rev. D. 86 (2012) 010001.

[14] W. Rodejohann, Int. J. Mod. Phys. E 20 (2011) 1833.

[15] M. Mitra, G. Senjanovic, F. Vissani, Nucl. Phys. B 856 (2012) 26;

[16] S. M. Bilenky, C. Giunti, Mod. Phys. Lett. A 27 (2012) 1230015,arXiv:1203.5250;

[17] W. Rodejohann, J. Phys. G 39 (2012) 124008, arXiv:1206.2560 [hep-ph].

[18] A. Merle, Int. J. Mod. Phys. D 22 (2013) 1330020. 